\title{
artigo
}

\section{Conhecimento da Oximetria de Pulso na Triagem da Cardiopatia Congênita}

\author{
Pulse Oximetry Knowledge in Congenital Cardiopathy Trial \\ Conocimiento de la oximetría de pulso en la detección de enfermedades cardíacas congénitas
}

\begin{abstract}
RESUMO
Objetivo: Fazer um levantamento sobre o conhecimento dos profissionais da saúde, nos hospitais, e graduandos dos cursos de Medicina e Enfermagem, relacionados com a aplicação da oximetria de pulso na triagem neonatal de cardiopatia congênita no Noroeste Paulista. Material e Método: Trata-se de um estudo transversal, de campo, quantitativo, no período de março a dezembro/2017. A amostra contou com 90 participantes distribuídos em três grupos: 55 graduandos de Medicina; 28 graduandos de Enfermagem e sete Profissionais/ Hospitais e participação de sete hospitais. A coleta de dados deu-se por meio de um questionário com treze questões, respondido pelos voluntários. Os dados foram planilhados por meio dos programas Microsoft Office Excel, Word e o Statistical Package for Social Sciences (SPSS) versão/24.0 e utilizado a estatística descritiva. Resultados: Foi constatado que somente $63 \%$ dos hospitais participantes realizam a triagem neonatal. Com relação ao treinamento, $49 \%$ dos graduandos de Medicina e nenhum de Enfermagem passaram por treinamento. Entre os Profissionais que atendem nos hospitais, somente $28 \%$ receberam treinamento. Conclusão: Constatou-se a baixa adesão na realização da oximetria de pulso pelos hospitais públicos, com maternidade, dessa região; bem como o déficit de treinamento ofertado aos seus profissionais. Há necessidade de ampliar o conhecimento da oximetria de pulso para a comunidade acadêmica dos cursos de Medicina e Enfermagem, e ofertar treinamentos aos profissionais que atendem os recém-nascidos nos berçários.
\end{abstract}

DESCRITORES: Oximetria de Pulso; Triagem da Cardiopatia Congênita; Teste do Coraçãozinho.

\section{ABSTRACT}

Objective: Conducting a survey on healthcare providers knowledge, at hospitals, Nursing and Medical school undergraduate students, regarding to pulse oximetry application on congenital heart disease neonatal triage in Paulista Northwest. Material and Method: This is a cross-sectional field qualitative study, from March to December/2017. The sample counted on 90 participants distributed in three groups: 55 Medical undergraduate students; 28 Nursing undergraduate students, seven Practitioners/Hospitals and seven hospitals. Data collections were through a questionnaire with thirteen questions answered by volunteers. Data were organized in spreadsheet by using Microsoft Office Excel, Word and Statistical Package for Social Sciences (SPSS) version/24.0 and using descriptive statistics. Results: It was found that only $63 \%$ of the participating hospitals screened neonatal patients. Regarding to training, $49 \%$ of Medical undergraduate students and nether from Nursing school were trained. Among practitioners assisting hospitals, only $28 \%$ were trained. Conclusion: It was verified low adhesion to pulse oximetry application by public maternity hospitals, in this region; as well as lack of personnel training. There is the necessity to spread knowledge about pulse oximetry to either Medical and Nursing academic community and practitioners assisting newborn babies in nursery. DESCRIPTORS: Pulse Oximetry; Congenital Cardiopathy Trial; Little Heart Test.

\section{RESUMEN}

Objetivo: realizar una encuesta sobre el conocimiento de los profesionales de la salud, en hospitales y estudiantes universitarios de cursos de medicina y enfermería, relacionada con la aplicación de la oximetría de pulso en la detección neonatal de enfermedades cardíacas congénitas en el noroeste de São Paulo. Material y Método: Este es un estudio de campo transversal, cuantitativo, de marzo a diciembre / 2017. La muestra incluyó 90 participantes divididos en tres grupos: 55 estudiantes de medicina; 28 estudiantes de enfermería y siete profesionales / hospitales y la participación de siete hospitales. La recolección de datos se realizó a través de un cuestionario con trece preguntas, respondidas por los voluntarios. Los datos se difundieron utilizando Microsoft Office Excel, Word y el paquete estadístico para ciencias sociales (SPSS) versión / 24.0 y se utilizaron estadísticas descriptivas. Resultados: se encontró que solo el $63 \%$ de los hospitales participantes realizan pruebas de detección neonatales. Con respecto a la capacitación, el $49 \%$ de los estudiantes de medicina y ninguno en enfermería recibió capacitación. Entre los profesionales que trabajan en hospitales, solo el 28\% recibió capacitación. Conclusión: Hubo una baja adherencia en el desempeño de la oximetría de pulso en los hospitales públicos, con maternidad, en esta región; así como el déficit de formación ofrecido a sus profesionales. Existe la necesidad de ampliar el conocimiento de la oximetría de pulso a la comunidad académica de los cursos de Medicina y Enfermería, y ofrecer capacitación a profesionales que atienden a recién nacidos en guarderías.

DESCRIPTORES: Oximetría de Pulso; Detección de Enfermedades Cardíacas Congénitas; Teste del Corazoncito.

RECEBIDO EM: 27/01/2020 APROVADO EM: 27/01/2020 


\section{Márcia Staff Zanquetta}

Pediatra, Mestranda em Ciências da Saúde pela Faculdade de Medicina de Rio Preto - FAMERP, São José do Rio Preto/SP, Brasil. https://orcid.org/0000-0002-8688-3531

\section{Valquiria da Silva Lopes}

Enfermeira, Doutoranda em Ciências da Saúde pela Faculdade de Medicina de Rio Preto - FAMERP, São José do Rio Preto/SP, Brasil. https://orcid.org/0000-0001-9755-5798

\section{Moacir Fernandes de Godoy}

Médico, Doutor em Cirurgia Cardiovascular, Professor Adjunto da Faculdade de Medicina de São José do Rio Preto (FAMERP) no Departamento de Cardiologia e Cirurgia Cardiovascular. Livre-Docente em Cardiologia pela FAMERP, Brasil. https://orcid. org/0000-0001-8390-0933

\section{INTRODUÇÃO}

A oximetria de pulso é um método não invasivo para se monitorar de forma contínua ou não, a concentração de oxigênio que está sendo transportado pela hemoglobina que passa pelos capilares. Essa monitorização é feita, habitualmente, nas extremidades do corpo como lóbulo da orelha, ponta dos dedos e, nos recém-nascidos (RN), nas mãos e pés ${ }^{(1)}$, e para realizar essa aferição utiliza-se um aparelho chamado oxímetro.

A concentração de oxigênio mensurada com o oxímetro é chamada de nível de saturação de oxigênio - SapO2 $2^{(2,3)}$. Este método tem sido utilizado por vários países, como um método de triagem para cardiopatias congênitas críticas (CCC), pois se trata de um método rápido, fácil, indolor, barato e de boa aceitação pelos responsáveis pelo $\mathrm{RN}^{(4-6)}$.

As CCC são malformações na estrutura anatômica do coração e dos grandes vasos que ocorrem ainda no período embrionário (período crítico da cardiogênese). $O$ período da cardiogênese se dá na metade da terceira semana ao final da oitava semana de gestação( ${ }^{(7)}$. São conhecidas como CCC as cardiopatias canal dependentes (que dependem da patência do canal arterial), e levam a descompensação hemodinâmica no período neonatal, podendo ocorrer nas primeiras horas ou primeiros dias de vida do RN, com elevada morbimortalidade, quando ocorre o fechamento do canal arterial ${ }^{(8-10)}$.

No mundo, a prevalência da cardio-
No mundo, a

prevalência da

cardiopatia

congênita é de 9,1

por 1.000 nascidos

vivos, no Brasil, 8

por 1.000 nascidos

vivos, sendo que as

CCC representam

2 por 1.000

nascidos vivos,

provocando elevada

morbimortalidade

no período neonatal

$\operatorname{precoce}^{(11,12)}$. patia congênita é de 9,1 por 1.000 nascidos vivos, no Brasil, 8 por 1.000 nascidos vivos, sendo que as CCC representam 2 por 1.000 nascidos vivos, provocando elevada morbimortalidade no período neonatal precoce ${ }^{(11,12)}$. O diagnóstico oportuno é importante para o prognóstico desses RN, porém os métodos atuais de rastreamento podem perder até 50\% dos $\mathrm{RN}$ afetados antes do nascimento, e os que são enviados para casa antes do diagnóstico frequentemente morrem ou sofrem uma grande morbidade ${ }^{(13)}$.

Neste sentido, é importante observar que uma característica de algumas CCC é a hipoxemia e/ou a diferença de saturação entre membro superior pré-ductal e membro inferior pós-ductal, que pode ser aferida com o uso do oxímetro ${ }^{(9)}$.

Após o nascimento, estruturas do sistema circulatório fetal (placenta, vasos umbilicais, ducto venoso, forame oval e canal arterial) deixam de existir e se estabelece a circulação neonatal. O canal arterial, por exemplo, tem seu fechamento funcional entre 12 e 15 horas de vida extrauterina, e o fechamento anatômico entre o quinto e sétimo dia, podendo chegar até 21 dias em alguns $\mathrm{RN}^{(12)}$.

Dessa maneira, a patência do canal arterial é que possibilita a sobrevida desses RN com CCC, por isso, a necessidade de se aproveitar essa "janela", para um diagnóstico rápido e preciso, para se manter esse canal arterial patente, até que se estabeleça a correção definitiva da cardiopatia ${ }^{(8)}$. Para manter-se a patência do canal arterial utiliza-se a Prostaglandina E2 - PGE2 (um fármaco que atua na musculatura lisa do vaso sanguíneo 


\section{artigo}

promovendo seu relaxamento). Com a finalidade de possibilitar um diagnóstico rápido é que se realiza a triagem por meio da oximetria de pulso entre 24 a 48 horas de vida do RN, antes da alta hospitalar, e uma vez constatada a hipoxemia, esse RN deve ser submetido a um ecocardiograma para que se estabeleça o diagnóstico da $\mathrm{CCC}^{(5,6)}$.

No tocante, as evidências atuais apoiam a introdução de triagem de rotina em RN assintomáticos, antes da alta do berçário do RN. O teste parece viável em vários países de rendimento alto e médio e mostra uma precisão consistente. No Brasil este método de rastreio por meio da oximetria de pulso recebe o nome de "Teste do coraçãozinho" $\mathrm{TC}^{(5)}$

A triagem para CCC por meio do TC está regulamentada no Brasil pela a Lei n. ${ }^{\circ} 4.163 / 13$, desde 21 de dezembro de 2011, e sua realização se tornou um procedimento nacional nos serviços de saúde públicos, pelo Sistema Único de Saúde (SUS), desde 10 de junho de 2014, seguindo o fluxograma norteado pelo Ministério da Saúde conforme a Figura $1^{(2)}$, e nos serviços privados.

O fluxograma acima auxilia os profissionais da saúde na identificação precoce das CCC, uma vez que elas são as causas mais frequente de anomalias congênitas graves (principalmente no período neonatal precoce, de RN com zero a sete dias de vida), sendo considerada hoje um problema de saúde pública global $^{(13,14)}$.

Os estudos mostram a elevada especificidade de $99 \%$ e moderada sensibilidade $67 \%$ a $72 \%$ deste método do $\mathrm{TC}^{(4,6,8,16)}$. Por ter elevada especificidade e possibilitar o diagnóstico enquanto o doente ainda esteja assintomático, sem descompensação hemodinâmica, todos esses fatores fazem com que o TC seja um bom método de triagem para $\mathrm{CCC}^{(10)}$, podendo ser realizado por médicos e profissionais da saúde, tal como equipe de enfermagem (Enfermeiros e

Figura 1. Fluxograma para triagem neonatal de cardiopatia congênita crítica. São José do Rio Preto, SP, Brasil, 2017

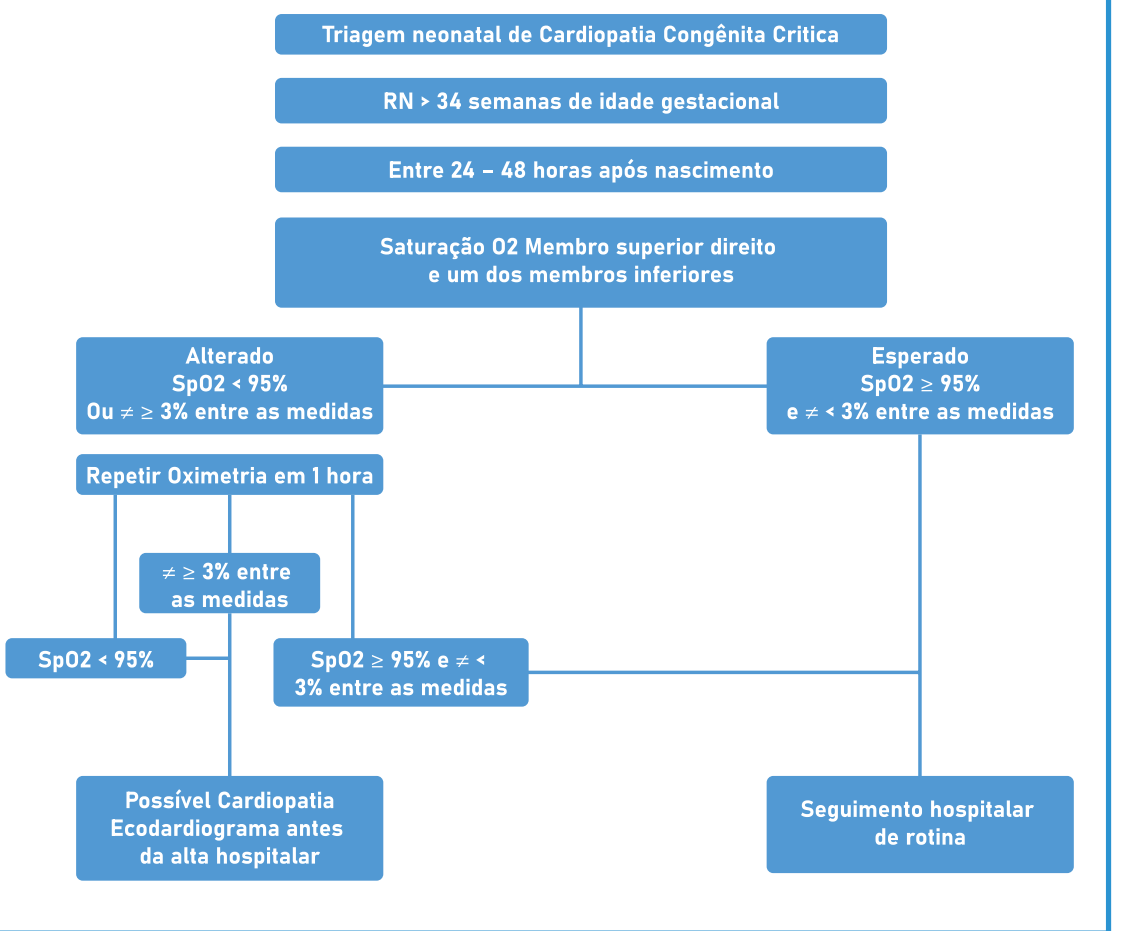

Nota: $\mathrm{RN}$ : recém-nascido; $\mathrm{SpO}_{2}$ : saturação de oxigênio.
Técnicos de Enfermagem), desde que tenham qualificação, conhecimento, capacitação e treinamento para sua realização ${ }^{(4,10,16,17)}$.

Desta forma, o presente estudo tem como por finalidade fazer um levantamento sobre o conhecimento da aplicação do TC como rastreio para algumas CCC, pela equipe de saúde hospitalar (Pediatras, Enfermeiros e Técnicos de Enfermagem) dos hospitais da região Noroeste Paulista, e graduandos do último ano de Medicina e Enfermagem de uma instituição universitária pública do Noroeste Paulista, de Santa Fé do Sul a São José do Rio Preto, que realizam a triagem por meio do TC em seus serviços.

Objetivou-se, com base no exposto, fazer um levantamento sobre o conhecimento dos profissionais da saúde, nos hospitais públicos com maternidade, e graduandos dos cursos de Medicina e Enfermagem, do Noroeste Paulista, relacionados com a aplicação do TC na triagem de CCC.

\section{METODOLOGIA}

Trata-se de um estudo de campo, transversal, quantitativo, realizado no período de março a dezembro de 2017. $\mathrm{O}$ estudo foi aprovado no Comitê de Ética de Pesquisa (CEP) com seres humanos da Faculdade de Medicina de Rio Preto (FAMERP), conforme a Resolução n. ${ }^{\circ}$ 466/2012 do Conselho Nacional de Saúde (CNS), com o CAAE 56675116.0.0000.5415 e parecer n. ${ }^{\circ}$ 1.646.502. Todos voluntários convidados a participarem do presente estudo assinaram o Termo de Consentimento Livre e Esclarecido (TCLE) para posterior utilização dos dados obtidos e a confidencialidade dos dados coletados, garantindo o anonimato dos envolvidos.

A amostra contou com 90 voluntários divididos em três grupos $(\mathrm{G})$, assim denominados: (G1) graduandos do último ano de Medicina; (G2) graduandos do último ano de Enfermagem; (G3) profissionais responsáveis pela aplicação TC nos berçários dos hospitais partici- 
pantes (hospitais públicos com maternidade no Noroeste Paulista de Santa Fé do Sul a São José do Rio Preto). A amostra foi obtida de forma aleatória, de acordo com a aceitação dos voluntários em participar da pesquisa.

Para os profissionais da saúde (Médicos, Enfermeiros, Técnicos de Enfermagem), o critério adotado foi ter mais de seis meses de experiência na prática da realização do TC. Para os graduandos dos cursos de Medicina e Enfermagem que estivessem cursando o último ano, por supostamente, já terem conhecimento prévio da aplicação do TC, e os hospitais públicos, com maternidade, localizados entre Santa Fé do Sul a São José do Rio Preto/SP, perfazendo uma área percorrida de $210 \mathrm{Km}$.

$O$ recurso utilizado para o levantamento de dados da pesquisa foi um questionário, contendo treze questões, dentre as quais três questões versavam sobre os dados demográficos: faixa etária, gênero, tempo de profissão (estudo/estágio) e área de atuação (estudo/estágio); Sete questões específicas relacionadas ao conhecimento sobre o TC: 1 - Você sabe o que é o Teste do Coraçãozinho (TC) ?; 2 - O que o TC avalia?; 3 - Você passou por treinamento para fazer o TC?; 4 - Com quantas horas de vida do recém-nascido você faz o TC?; 5 - Se for preciso repetir o TC com quanto tempo você o refaz?; 6 - Aonde é colocado o sensor do oxímetro?; 7 - Se após repetir em 1 hora o TC continuar alterado, a conduta é?; Três questôes relacionadas à realidade das instituições participantes (hospitais): 1 - Neste hospital tem cardiopediatra?; 2 - Em caso de transferência do recém-nascido, quantas horas em média, leva a transferência?; 3 - Quantos partos mensais são realizados, em média, nesse hospital?

O coordenador do curso de Medicina autorizou aplicar o questionário por grupos e individualmente nos locais de atuação que os graduandos de Medicina estavam fazendo estágio, após autorização prévia do preceptor responsável pelo estágio local. Quanto aos graduandos de Enfermagem, o questionário foi aplicado após agendamento com a coordenação do curso de Enfermagem, em um dia de prova, onde toda turma do último ano estava presente, quando responderam ao questionário individualmente.

\section{Para os}

\section{profissionais da}

saúde (Médicos,

Enfermeiros,

\section{Técnicos de}

\section{Enfermagem), o \\ critério adotado}

foi ter mais de

seis meses de

experiência

na prática da

realização

do TC.

Para aplicar o questionário nos hospitais, foi enviada uma carta ao Diretor Técnico dos Hospitais participantes solicitando autorização para acesso ao local. Sendo feito contato prévio para agendamento com o médico responsável pelo setor da Pediatria/Neonatologia para que autorizasse a aplicação do questionário aos funcionários que realizavam o TC, em um dos períodos do plantão (sendo consentida a visita no período vespertino). Todos os participantes assinaram o TCLE.

A pesquisadora aguardou junto aos voluntários o preenchimento do questionário. Os graduandos do curso de Medicina ingressam na especialidade Pediatria, segundo o plano de ensino dessa instituição, no terceiro ano com carga horária de 45 horas; no quarto ano Pediatria e Puericultura com carga horária de 75 horas e no quinto ano com carga horária de Pediatria 257 horas, mais estágio na especialidade de 88 horas, perfazendo um total de 345 horas. No sexto ano a carga horária na disciplina é de 180 horas para estágio de 66 horas para plantão. As aulas sobre triagem neonatal são ministradas no quinto ano.

Os graduandos de Enfermagem têm acesso a um breve conteúdo sobre o TC no plano de ensino na disciplina de Políticas de Saúde, estando o tema “Triagem Neonatal" também inserido no plano de ensino da disciplina de "Saúde da Criança e do Adolescente". Disciplinas ministradas no terceiro ano da graduação, duração de um semestre, carga horária total (225/270 horas), teoria (60 a 72 horas), prática (165 a 198 horas). Os alunos ainda participam de estágios nas disciplinas de Saúde da Criança e Saúde da Mulher em vários cenários, um desses cenários é no Alojamento Conjunto, do hospital escola da instituição que estudam.

Os dados foram organizados em planilha por meio dos programas Microsoft Office Excel, Word e o Statisctical Package for Social Sciences (SPSS), versão 24.0. Para análise estatística dos resultados utilizou-se a estatística descritiva absoluta e relativa. Os resultados foram expostos em tabelas. 
Tabela 1. Dados sociodemográficos dos idosos participantes do estudo. Álvares Machado, SP, Brasil, 2016.

Variáveis

G1
N

$(\%)$

G2

G3

\section{Gênero}

Feminino

Masculino

Total

Faixa etária

$18-24$ anos

25 - 34 anos

35 - 44 anos

Acima de 45 anos

Total

Tempo de atuação

6 meses a 1 ano

1 a 5 anos

5 a 10 anos

Acima de 10 anos

Estudo/Estágio

Não respondeu

Total

Nota: G: grupo; N: número; \%: porcentagem.

55

$\begin{array}{cccccc}36 & 65,45 \% & 23 & 82,14 \% & 5 & 71,42 \% \\ 19 & 34,55 \% & 5 & 17,86 \% & 2 & 28,58 \% \\ 55 & 100 \% & 28 & 100 \% & 7 & 100 \%\end{array}$

$\begin{array}{cccccc}30 & 54,5 \% & 27 & 96,4 \% & 0 & 0 \% \\ 23 & 41,8 \% & 1 & 3,6 \% & 2 & 28,6 \% \\ 2 & 3,6 \% & 0 & 0 \% & 2 & 28,6 \% \\ 0 & 0 \% & 0 & 0 \% & 3 & 43,0 \% \\ 55 & 100 \% & 28 & 100 \% & 7 & 100 \%\end{array}$

Tabela 2. Percepção por grupos dos graduandos e profissionais da saúde frente ao TC. São José do Rio Preto, SP, Brasil, 2017

\section{Perguntas do conhecimento da aplicação do Teste do Coraçãozinho}

1. Você sabe o que é o TC?

2. 0 que o TC avalia?

3. Você passou por treinamento para fazer o TC?

4. Com quantas horas de vida o RN, você faz o TC?

5. Se for preciso repetir o TC, com quantas horas você o refaz?

6. Se após repetir o TC e continuar alterado, a conduta é?

7. No hospital que você trabalha ou faz estágio tem cardiopediatra?

Médiageral deacerto frenteao TC

Nota: TC: teste do coraçãozinho; G: grupo;

\section{Respostas}

G1

G2

$3,7 \% \quad 1 \quad 14,3 \%$

$0 \% \quad 1 \quad 14,3 \%$

$0 \% \quad 1 \quad 14,3 \%$

$3,7 \% \quad 4 \quad 57,1 \%$

$92,6 \% \quad 0 \quad 0 \%$

$3,7 \% \quad 0 \quad 0 \%$ $100 \% \quad 7 \quad 100 \%$

$\%$


mesmo que regulamentado em portarias do Ministério da Saúde com a implantação do TC de forma universal pelo SUS, no Brasil ${ }^{(5,13,14)}$. O TC, no Brasil, veio se somar a sua utilização evidenciada em outros países ${ }^{(6,8-10)}$, mostrando uma boa taxa de sensibilidade $(75-76,5 \%)$ e alta especificidade $(99,99 \%)$ para a $\operatorname{CCC}^{(8,10)}$.

A triagem pode ser realizada pelo pediatra e pela equipe de enfermagem, desde que conheçam a técnica corretamente, saibam manipular o aparelho para sua realização e saibam o que está sendo avaliado $^{(8,16)}$. Diversos estudos ${ }^{(9,16-20)}$ deixam clara a necessidade da realização da triagem por pessoal qualificado, treinado no uso do algoritmo, e na monitorização da oximetria de pulso em RN. A sensibilidade e fiabilidade da triagem podem ser influenciadas por fatores humanos, e o nível de conhecimento do pessoal ${ }^{(18)}$.

Neste estudo, observou-se que há grande necessidade de treinamento dos profissionais de saúde que trabalham em hospitais públicos, com maternidade, e que realizam testes de triagem neonatal, em especial o TC. Um outro estudo mostra que $77 \%$ dos enfermeiros não conheciam ou não tinham ouvido falar do TC, antes de sua implantação na unidade hospitalar ${ }^{(16)}$, o que vem consolidar os dados levantados neste estudo (G2 - 43\% dos que responderam as questões).

Estudos demonstram que a compreensão sobre o TC por profissionais de saúde (Médicos e equipe de Enfermagem), que lidam rotineiramente com cuidados de saúde, é ainda muito deficiente, podendo levar a desfechos indesejáveis ${ }^{(21)}$. Estudos futuros são necessários para se avaliar a implantação deste método de triagem nos hospitais brasileiros, e a capacitação

\section{O curso de}

Enfermagem

apresentou baixo

conhecimento

quanto à aplicação

do $\mathrm{TC}$, em relação

à Medicina e aos

profissionais da

saúde, mesmo que

regulamentado

em portarias do

Ministério da Saúde

com a implantação

do TC de forma

universal pelo SUS,

no Brasil ${ }^{(5,13,14)}$. dos profissionais que realizam o $\mathrm{TC}$ em outros estados do país.

Os estudos científicos ${ }^{(16,19,21)}$ deixam claro a carência de treinamento, conhecimento e interpretação desses profissionais (Médicos e equipe de Enfermagem, bem como os graduandos de Medicina e Enfermagem), e a necessidade de educação continuada para aprofundar o conhecimento amplo do uso dessa ferramenta, que se mal interpretada pode onerar ainda mais o sistema público de saúde em nosso país, o SUS, e acarretar danos irreversíveis aos recém-nascidos que passaram pela triagem de forma mal interpretada ${ }^{(22)}$.

\section{CONCLUSÃO}

Verificou-se baixa adesão na realização do TC pelos hospitais públicos com maternidade do Noroeste Paulista; bem como a falta de treinamento e capacitação ofertados aos profissionais que trabalham nessas instituições, e aos graduandos de Medicina e Enfermagem. Propõem-se um olhar crítico das instituições de ensino que formam esses profissionais, e uma reavaliação de sua grade curricular. As Sociedades de Pediatria estaduais e Enfermagem podem contribuir com programas de educação continuada e capacitação dos profissionais que se encontram em áreas mais afastadas das grandes cidades.

\section{AGRADECIMENTOS}

Agradecemos os graduandos e profissionais da saúde que doaram um pouco de seu tempo em prol de nossa pesquisa, bem como as instituições que nos receberam.

\section{REFERÊNCIAS}

1. Hinkelbein J, Genzwuerker HV, SogI R, Fiedler F. Effect of nail polish on oxygen saturation determined by pulse oximetry in critically ill patients. Resuscitation. [Internet]. 2007[cited $2017 \mathrm{dez} 18] ; 72(1): 82-91$. Available from: https://www. ncbi.nlm.nih.gov/pubmed/17098347.
2. Sinex JE. Pulse oximetry: principles and limitations. Am J Emerg Med. [Internet]. 1999 [cited 2017 dez 18];17(1): 59-67. Available from: https://www.ncbi.nlm.nih.gov/pubmed/9928703.

3. Severinghaus JW. Takuo Aoyagi: discovery of pulse oximetry. 


\section{REFERÊNCIAS}

Anesth Analg. [Internet]. 2007[cited 2017 dez 18];105(6Suppl):S1-4. Available from: https://www.ncbi.nlm.nih.gov/pubmed/18048890.

4. Mahle WT, Martin GR, Beekman RH, Morrow WR, Rosenthal GL, Snyder CS, et al. Endorsement of Health and Human Services recommendation for pulse oximetry screening for critical congenital heart disease. Pediatrics. [Internet]. 2012 Jan 1 [cited 2017 dez 18];129(1):190-2. Available from: https:// www.ncbi.nlm.nih.gov/pubmed/22201143.

5. SOCIEDADE BRASILEIRA DE PEDIATRIA. Teste do Coraçãozinho. Departamentos Científicos de Cardiologia e Neonatologia da SBP. [Internet]. [cited 2017 jul 24]. Available from: https://www.sbp.com.br/fileadmin/user_upload/2015/02/ diagnostico-precoce-oximetria.pdf.

6. Thangaratinam S, Brown K, Zamora J, Khan KS, Ewer AK. Pulse oximetry screening for critical congenital heart defects in asymptomatic newborn babies: a systematic review and meta-analysis. The Lancet. [Internet]. 2012 Jun 30. [cited 2017 dez 18];379(9835):2459-64. Available from: https:// www.ncbi.nlm.nih.gov/pubmed/22554860.

7. Sadler TW. Langman embriologia médica. 12. ed. Rio de Janeiro: Guanabara Koogan; 2013.

8. Mahle WT, Newburger JW, Matherne GP, Smith FC, Hoke TR, Koppel R, et al. Role of pulse oximetry in examining newborns for congenital heart disease: a scientific statement from the American Heart Association and American Academy of Pediatrics. Circulation. [Internet]. 2009 Aug 4.[cited 2017 dez 18];120(5):447-58. Available from: https://www.ncbi.nlm.nih. gov/pubmed/19581492.

9. Granelli AD, Wennergren M, Sandberg K, Mellander M, Bejlum C, Inganäs L, et al. Impact of pulse oximetry screening on the detection of duct dependent congenital heart disease: a Swedish prospective screening study in 39821 newborns. Bmj. [Internet]. 2009 Jan 9. [cited 2017 dez 18];338:a3037. Available from: https://www.bmj.com/content/338/bmj. a3037.

10. Du C, Liu D, Liu G, Wang H. A Meta-Analysis about the Screening Role of Pulse Oximetry for Congenital Heart Disease. BioMed research international. [Internet]. 2017. [cited 2017 dez 18]. Available from: https://www.ncbi.nlm.nih.gov/ pubmed/29376068.

11. Ministério da Saúde (BR). Comissão Nacional de Incorporação de Tecnologias no SUS (CONITEC) - Relatório n. ${ }^{\circ} 115$. Teste do coraçãozinho (Oximetria de Pulso) na triagem neonatal. Brasília, DF: MS; 2015.

12. Vander LD, Konings EE, Slager MA, Witsenburg M, Helbing WA, Takkenberg JJ, et al. Birth prevalence of congenital heart disease worldwide: a systematic review and meta-analysis. Journal of the American College of Cardiology. [Internet] 2011 Nov 15. [cited $2017 \mathrm{dez} 18] ; 58(21): 2241-7$. Available from: https://www.ncbi.nlm.nih.gov/pubmed/22078432.

13. Sendelbach DM, Jackson GL, Lai SS, Fixler DE, Stehel EK,
Engle WD. Pulse oximetry screening at 4 hours of age to detect critical congenital heart defects. Pediatrics. [Internet]. 2008 [cited 2017 dez 18];122(4):e815 20. Available from: https://www.ncbi.nlm.nih.gov/pubmed/18762486.

14. Mattos SS. Fisiologia da Circulação Fetal e Diagnóstico das Alterações Funcionais do Coração do Feto, Recife, PE. Arq. Bras. Cardiol. [Internet]. 1997. [cited 2017 dez 18]; 69(3). Available from: http://www.scielo.br/scielo.php?scrip$\mathrm{t}=\mathrm{sci}$ _arttext\&pid=S0066-782X1997000900013.

15. Ministério da Saúde, Secretaria de Atenção à Saúde (BR). Aprova o Plano Nacional de Assistência à Criança com Cardiopatia Congênita. Portaria n. ${ }^{\circ} 1.727$, De 11 de Julho De 2017. Brasília (DF); MS; 2017.

16. Medeiros AL, Bezerra FT, Sousa SAJ, da Silva MS. Oximetria de pulso em triagem de cardiopatias congênitas: conhecimento e atuação do enfermeiro. Cogitare Enfermagem. [Internet]. 2015[cited 2017 dez 18];20(3). Available from: http://docs. bvsalud.org/biblioref/2016/08/1237/40941-163352-1-pb. pdf.

17. Milutinović D, Repić G, Aranđelović B. Clinical nurses' knowledge level on pulse oximetry: A descriptive multi-centre study. Intensive and Critical Care Nursing. [Internet]. 2016 Dec 1.[cited 2017 dez 18];37:19-26. Available from: https:// www.ncbi.nlm.nih.gov/pubmed/27575619.

18. Seeley MC, Mckenna L, Hood K. Graduate nurses' knowledge of the functions and limitations of pulse oximetry. Journal of clinical nursing. [Internet]. 2015.[cited 2017 dez 18];24(23-24):3538-49. Available from: https://www.ncbi. nlm.nih.gov/pubmed/26419943.

19. Ferreira ML, da Silva CM, Reis JM, da Silva CM. $O$ teste de triagem neonatal de cardiopatias congênitas: uma tecnologia de cuidado de enfermagem. Academus Revista Científica da Saúde. [Internet]. 2016 Apr 4[cited 2017 dez 18];1(1). Available from: https://smsrio.org/revista/index.php/reva/article/ view/131.

20. Morais S, Mimoso G. Oximetria de pulso no diagnóstico de cardiopatia congénita. Sugestões para a implementação de uma estratégia de rastreio. Acta Pediátrica Portuguesa. [Internet]. 2014. [cited $2017 \mathrm{dez} 18] ; 44(6)$. Available from: https://core.ac.uk/download/pdf/61498468.pdf.

21. Fouzas S, Politis P, Skylogianni E, Syriopoulou T, Priftis KN. Chatzimichael A. Knowledge on pulse oximetry among pediatric health care professionals: a multicenter survey. Pediatrics. [Internet]. 2010[cited 2017 dez 18]: peds. 20100849. Available from: https://www.ncbi.nlm.nih.gov/pubmed/20679299.

22. Lacerda LF de, Ferreira ALC, Lisboa CB, Lúcio IML, Batista JCL, Melo LO. Triagem neonatal de cardiopatias congênitas: percepção dos profissionais de saúde do alojamento conjunto. Rev enferm UFPE on line., Recife, [Internet]. 2016. jul. [cited $2017 \mathrm{dez} 18] ; 10(7): 2420-7$. Available from: https://periodicos.ufpe.br/revistas/revistaenfermagem/article/viewFile/11298/12962. 